\title{
Conformational Dynamics of Transmembrane Domain 3 of Presenilin 1 Is Associated with the Trimming Activity of $\gamma$-Secretase
}

\author{
ㅈTtetsuo Cai, ${ }^{1}$ Kanan Morishima, ${ }^{2}$ Shizuka Takagi-Niidome, ${ }^{1}$ Aya Tominaga, ${ }^{1}$ and ${ }^{\circ}$ Taisuke Tomita ${ }^{1}$ \\ ${ }^{1}$ Laboratory of Neuropathology and Neuroscience, Graduate School of Pharmaceutical Sciences, and ${ }^{2}$ Laboratory of Neuropathology and Neuroscience, \\ Faculty of Pharmaceutical Sciences, University of Tokyo, Bunkyo-ku, Tokyo 113-0033, Japan
}

$\gamma$-Secretase is an intramembrane-cleaving protease that generates the toxic species of the amyloid- $\beta$ peptide $(\mathrm{A} \beta)$ that is responsible for the pathology of Alzheimer disease. The catalytic subunit of $\gamma$-secretase is presenilin 1 (PS1), which is a polytopic membrane protein with a hydrophilic catalytic pore. The length of the $C$ terminus of $A \beta$ is proteolytically determined by its processive trimming by $\gamma$-secretase, although the precise mechanism still remains largely unknown. Here, we identified that transmembrane domain (TMD) 3 of human PS1 is involved in the formation of the intramembranous hydrophilic pore. Notably, the water accessibility of TMD3 was greatly altered by point mutations and compounds, which modify $\gamma$-secretase activity. The changes in the water accessibility of TMD3 was also correlated with $\mathrm{A} \beta 42$ production. Moreover, crosslinking between TMD 3 and TMD7 resulted in a loss of sensitivity to a $\gamma$-secretase modulator that reduces $\mathrm{A} \beta 42$ production. Therefore, our findings indicate that the conformational dynamics of TMD 3 is a prerequisite for regulation of the $\mathrm{A} \beta$ trimming activity of $\gamma$-secretase.

Key words: Alzheimer; amyloid; enzyme; membrane; secretase; structure

\section{Significance Statement}

Modulation of $\gamma$-secretase activity to reduce the level of toxic amyloid- $\beta$ species is thought to be a therapeutic strategy for Alzheimer disease. However, the detailed mechanism of the regulation of amyloid- $\beta$ production, as well as the structure-andactivity relationship of $\gamma$-secretase remains unclear. Here we identified that the water accessibility around transmembrane domain 3 in presenilin 1 was increased along with a reduction in toxic amyloid- $\beta$ production. Our findings demonstrate how the structure of presenilin 1 dynamically changes during amyloid- $\beta$ production, and provides insights toward the development of treatments against Alzheimer disease.

\section{Introduction}

Alzheimer disease (AD) is the most common form of dementia. The main pathological feature of $\mathrm{AD}$ is the accumulation of senile plaques, which are mainly composed of amyloid- $\beta$ peptide

Received April 6, 2019; revised Aug. 16, 2019; accepted Aug. 30, 2019.

Author contributions: T.C., K.M., S.T.-N., A.T., and T.T. designed research; T.C., K.M., and S.T.-N. performed research; T.C. analyzed data; T.C. wrote the first draft of the paper; T.C., K.M., S.T.-N., A.T., and T.T. edited the paper; T.C. and T.T. wrote the paper.

This work was supported by Japan Society for the Promotion of Science Grants-in-Aid for Scientific Research (A) 15 H02492 and 19H01015 and Research Fellowship for Young Scientists 18J14653, Tokyo Biochemical Research Foundation, Nagase Science Technology Foundation, and Mitsubishi Foundation. We thank Drs. Bart De Strooper (Catholic University of Leuven, Leuven, Belgium), Toshio Kitamura (University of Tokyo, Tokyo), Gopal Thinakaran (University of Chicago, Chicago), Masahiro Yonaga (Eisai Company, Ltd., Ibaraki, Japan), Satoshi Yokoshima, and Tohru Fukuyama (Nagoya University, Nagoya, Japan) for valuable reagents; and our laboratory members for helpful discussions and technical assistance.

The authors declare no competing financial interests.

Correspondence should be addressed to Taisuke Tomita at taisuke@mol.f.u-tokyo.ac.jp.

https://doi.org/10.1523/JNEUROSCI.0838-19.2019

Copyright $\odot 2019$ the authors
$(\mathrm{A} \beta) . \mathrm{A} \beta$ is generated from amyloid precursor protein (APP) by its sequential cleavage by $\beta$-secretase and $\gamma$-secretase (Tomita, 2014). $\gamma$-Secretase successively cleaves every three or four residues, resulting in the generation of various species of $\mathrm{A} \beta$ with C-terminal lengths ranging from 43 to 37 (Qi-Takahara et al., 2005; Takami et al., 2009). Among these species, $A \beta 42$ has been shown to be the most toxic and aggregation-prone species that is predominantly deposited in the brains of $\mathrm{AD}$ patients (Iwatsubo et al., 1994). Thus, a deep understanding of the molecular mechanism by which $\gamma$-secretase cleaves $A \beta 42$ is required for the further development of efficient drugs against AD. $\gamma$-Secretase is an intramembrane protease comprising the following four subunits: presenilin (PS), nicastrin, anterior pharynx-defective 1, and presenilin enhancer 2 (Takasugi et al., 2003). PS is an aspartic protease subunit that shows both proteolytic and autoproteolytic activity to generate $\mathrm{N}$ - and C-terminal fragments (NTF and CTF). Recently, the cryo-electron microscopy (cryo-EM) structure of human $\gamma$-secretase was clarified (Bai et al., 2015). Al- 


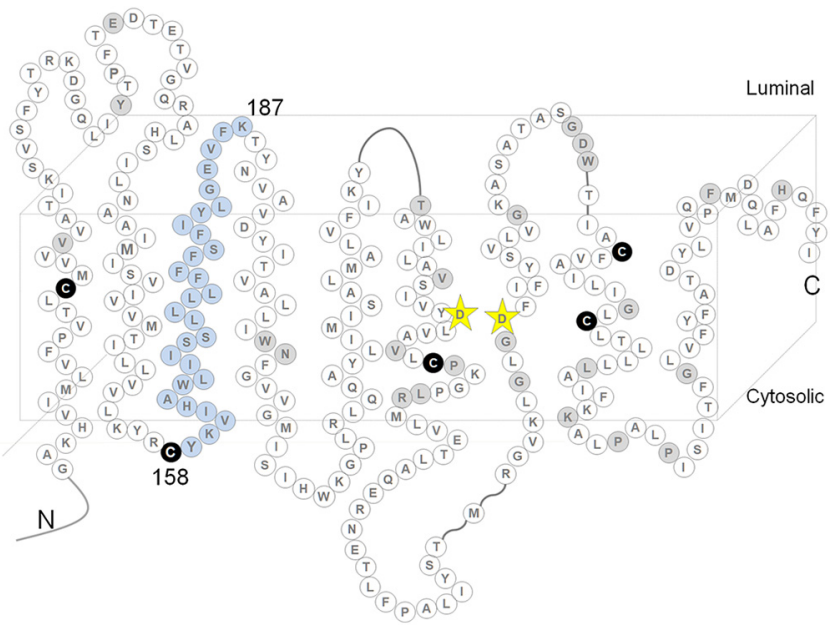

Figure 1. Locations of the Cys mutations of PS1 analyzed in this study. Black circles represent endogenous Cys that were replaced with Ser in PS1/Cys (-). Blue circles represent amino acid residues analyzed in this study. Gray circles represent residues in which Cys substitution resulted in a loss of $\gamma$-secretase activity. Yellow stars indicate the catalytic aspartates. from Psen1/Psen2 double KO (DKO) mice of either sex (Herreman et al., 2000); retroviral infections (Kitamura et al., 2003) were performed as described previously (Watanabe et al., 2005, 2010; Sato et al., 2006, 2008). For DKO cells coexpressing both PS1wt and FLAG-Pen-2 (Isoo et al., 2007), cells were incubated with half of each virus medium so that the total amount of medium was kept at the same. Immunoblotting was performed as described previously (Tomita et al., 1997; Morohashi et al., 2006; Sato et al., 2006).

Preparation of microsomes. Microsomes were prepared as described previously with some modifications (Morohashi et al., 2006; Sato et al., 2006; Tomita, 2017). Briefly, cell pellets were homogenized in homogenization buffer [20 mм HEPES, $140 \mathrm{~mm} \mathrm{KCl,} 0.25$ м sucrose, and 1 mM EGTA with complete protease inhibitor mixture (Roche Applied Science)] using an HG 30 homogenizer (Hitachi), and centrifuged at $1400 \times g$ for $10 \mathrm{~min}$ at $4^{\circ} \mathrm{C}($ OptimaTM L-90K Ultracentrifuge, Beckman Coulter). The supernatant was then centri-

though these reports have promoted our understating of the structure of $\gamma$-secretase, the structural dynamics of the enzyme remains unclear. We previously analyzed the structure of PS1 by the substituted cysteine accessibility method (SCAM) and crosslinking experiments (Tomita, 2017; Cai and Tomita, 2018). SCAM enables identification of the hydrophilic environment in a molecule via analysis of the accessibility of 3-(4,5-dimethylthiazol-2-yl)-5-(3-carboxymethoxyphenyl)-2-(4-sulfophenyl)2H-tetrazolium (MTS) reagents to cysteine residues (Cys) introduced into designed positions (Karlin and Akabas, 1998). Crosslinking experiments clarify the distance between two Cys at designed positions. Using combinations of these two methods, we clarified several structural features and the conformational dynamics of PS1, such as the catalytic pore, PAL motif, hydrophilic loop (HL), and transmembrane domains (TMDs) (Sato et al., 2006, 2008; Takagi et al., 2010; Takagi-Niidome et al., 2015; Tominaga et al., 2016; Cai et al., 2017; Imai et al., 2018). In this study, we focused on TMD3 of PS1, as several genetic studies have identified $\sim 30$ familial AD (FAD) mutations in TMD3, including the L166P mutation, which showed the strongest effect on the production of $A \beta 42$. A TMD-swapping study also suggested that TMD3 is not necessary for $\gamma$-secretase activity but is crucial for the regulation of $\mathrm{A} \beta 42$ production (Watanabe et al., 2010). Here we found that TMD3 is involved in the formation of the catalytic pore, and its conformational dynamics is important for the $\mathrm{A} \beta$ trimming activity of $\gamma$-secretase.

\section{Materials and Methods}

Antibodies and compounds. G1L3, which targets the hydrophilic loop 6 of PS1, was described previously (Tomita et al., 1999). G1Nr5 was raised against the $\mathrm{N}$ terminal of human PS1 protein (Sato et al., 2008). The anti-FALG tag antibody, monoclonal anti-FLAG M2, was purchased from Sigma-Aldrich. L-685,458 [(1S-benzyl-4R-[1-(1S-carbamoyl-2phenylethylcarbamoyl)-1S-3-methyl-butylcarbamoyl]-2R-hydroxy-5phenylpentyl) carbamic acid tert-butyl ester] (Shearman et al., 2000) was purchased from Bachem. E2012 was kindly provided from Dr. Yonaga (Esai). ST1120 was synthesized as previously reported (Takeo et al., 2014).

Plasmid construction, retrovirus infection, and immunological methods. cDNAs encoding human PS1 and APP carrying Swedish mutation (APPNL) were inserted into pMXs-puro (Kitamura et al., 2003). cDNAs encoding mutant PS1 were generated by long PCR-based QuikChange strategy (Stratagene). Maintenance of embryonic fibroblasts derived fuged at $29,000 \times g$ for $40 \mathrm{~min}$ at $4^{\circ} \mathrm{C}$. Pellets were collected and stored as microsome fractions at $-80^{\circ} \mathrm{C}$ until use. For preparation of solubilized microsomes, the microsome fraction was dissolved in solubilization buffer containing 1\% 3-[(3-cholamidopropyl) dimethylammonio]-2hydroxypropanesulfonate, $10 \mathrm{~mm} \mathrm{HEPES}$, and $150 \mathrm{~mm} \mathrm{NaCl}$ with complete protease inhibitor mixture, and centrifuged at $142,600 \times g$ for $60 \mathrm{~min}$ at $4^{\circ} \mathrm{C}$. The resulting supernatant was used immediately for analysis.

sC99-3 $\times$ FLAG purification. HEK293A cells overexpressing sC99$3 \times$ FLAG were first collected with DPBS and centrifuged at $750 \times g$ for 10 min at $4^{\circ} \mathrm{C}$. Then the pellets were dissolved in lysis buffer containing 50 mm Tris- $\mathrm{HCl}, \mathrm{pH} 7.4,150 \mathrm{~mm} \mathrm{NaCl}$, and $1 \%$ Nonidet P-40 with complete protease inhibitor mixture, rotated for $2 \mathrm{~h}$ at $4^{\circ} \mathrm{C}$. After rotation, the solution was centrifuged at $253,500 \times g$ for $20 \mathrm{~min}$ at $4^{\circ} \mathrm{C}$, and the resulting supernatant was gathered and rotated $\mathrm{O} / \mathrm{N}$ at $4^{\circ} \mathrm{C}$ with antiFLAG M2-agarose (Sigma-Aldrich). The next day, M2-agarose was washed 3 times by centrifuging at $335 \times \mathrm{g}$ for $5 \mathrm{~min}$ at $4^{\circ} \mathrm{C}$ with lysis buffer containing $50 \mathrm{~mm}$ Tris- $\mathrm{HCl}, \mathrm{pH} 7.4,150 \mathrm{~mm} \mathrm{NaCl}$, and $0.25 \%$ $n$-dodecyl- $\beta$-D-maltoside with complete protease inhibitor mixture. Then purified sC99-3×FLAG was eluted by $200 \mu$ lelution buffer containing $100 \mathrm{~mm}$ glycine, $\mathrm{pH} 2.7$, and $0.25 \% n$-dodecyl- $\beta$-D-maltoside, and rotated at room temperature for $10 \mathrm{~min}$. Soon after the rotation, the solution was centrifuged at $5700 \times g$ for $1 \mathrm{~min}$ at $4^{\circ} \mathrm{C}$, and $8 \mu \mathrm{l} 1 \mathrm{M}$ Tris- $\mathrm{HCl}, \mathrm{pH} 8.0$, was added to the resulting supernatant to neutralize the acid, used immediately for analysis.

SCAM and crosslinking experiments. SCAM experiments were performed as previously described (Cai and Tomita, 2018). Biotinylation and competition experiments using N-biotinoylaminoethyl methanethiosulfonate (MTSEA-biotin) in intact cells or microsome fractions have been described previously (Sato et al., 2006, 2008). For the competition assay, sodium 2-sulfonatoethyl methanethiosulfonate (MTSES), 2-(trimethylammonium) ethyl methanethiosulfonate bromide (MSTET), or 2-(triethylammonium)-ethyl methanethiosulfonate bromide (MTSTEAE) were preincubated with intact cells at $2 \mathrm{~mm}$ for $30 \mathrm{~min}$ or microsomes at $2 \mathrm{~mm}$ for $5 \mathrm{~min}$ at $4^{\circ} \mathrm{C}$ and washed once before biotinylation. Inhibitors were preincubated with intact cells for $30 \mathrm{~min}$ at $4^{\circ} \mathrm{C}$ before incubation for biotinylation at concentrations that completely abolish the proteolytic activity of $\gamma$-secretase (L-685,458, $1 \mu \mathrm{M}$ ) (Sato et al., 2006). $\gamma$-Secretase modulators were added in the culturing medium and preincubated with intact cells $1 \mathrm{~d}$ before conducting SCAM at an A $\beta 42$ lowering concentration (at $10 \mu \mathrm{M}$ ) (Takeo et al., 2014).

Crosslinking experiments were performed as described previously (Sato et al., 2006), except the following two: (1) for experiments using crosslinkers, microsomes were incubated for $30 \mathrm{~min}$ at $37^{\circ} \mathrm{C}$; and (2) for experiments using $\mathrm{CuSO}_{4}$ and phenanthroline $(\mathrm{Cu}-\mathrm{PNT})$ catalysts, the 
A
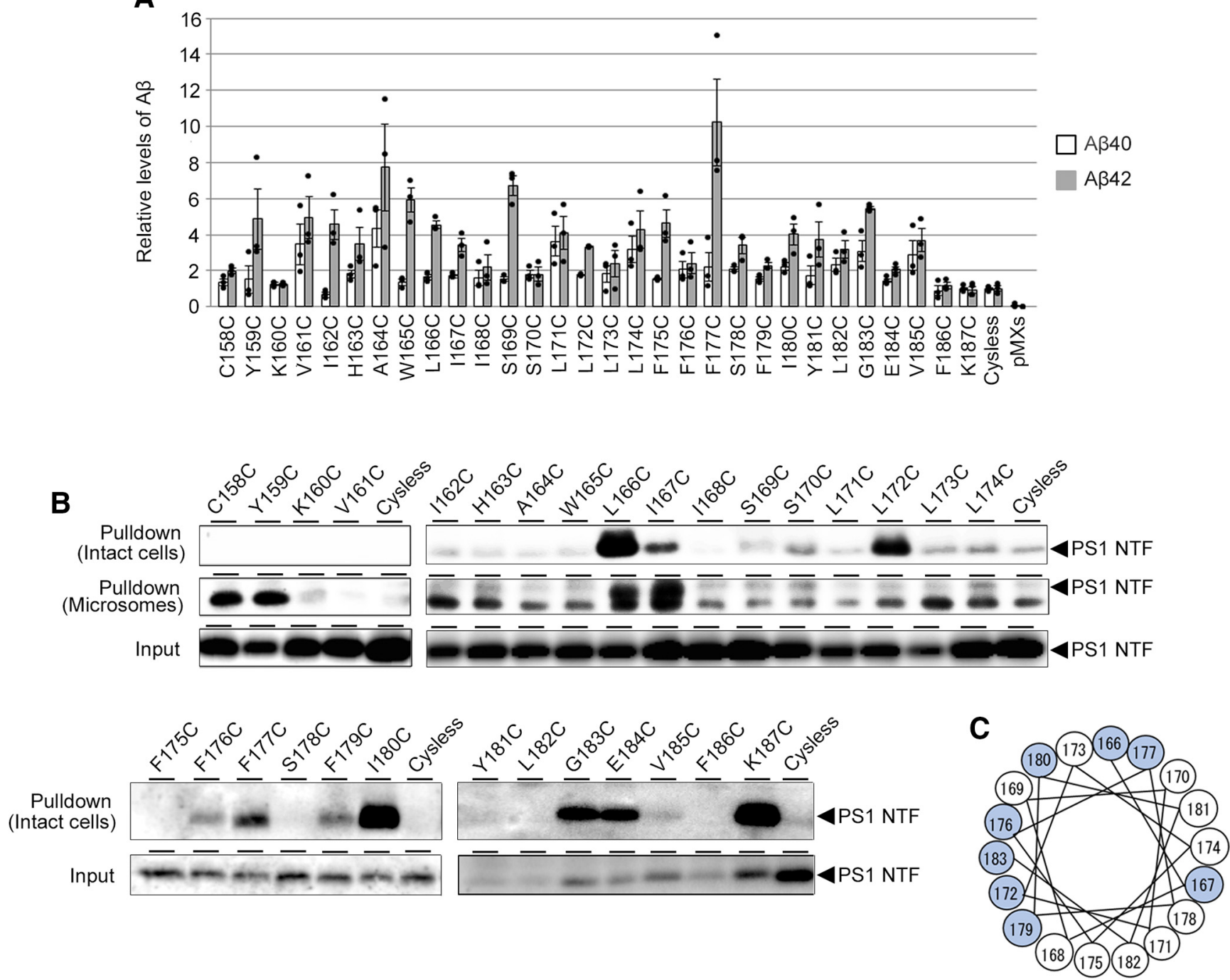

Figure 2. $\quad \gamma$-Secretase activity and SCAM analysis of single Cys mt PS1s mutated around TMD3. A, Sandwich ELISA of A $\beta$ secreted from APPNL-stable DKO cells transiently transfected with single-Cys mt PS1 ( $n=3$ or 4, mean \pm SEM). The levels of secreted A $\beta$ in conditioned media were normalized by that of cells expressing PS1/Cys ( - ). White bar represents production of A $\beta 40$. Gray bar represents production of A $\beta 42$. B, Biotinylation experiment using MTSEA-biotin in intact cells and microsomes. Immunoblotting assay was performed using the anti-PS1 NTF antibody G1Nr5. Amounts of PS1 NTF in the pulldown fraction from intact cells (top), the pulldown fraction from microsomes (middle), and the input fraction (bottom). C, The TMD3 helix model. Blue represents residues labeled by MTSEA-biotin. All except I167C localize on one side of the helix.

reagents were added at the final concentration of 3 and $15 \mathrm{~mm}$ each and were incubated at room temperature for $2 \mathrm{~h}$.

Sandwich ELISA. For the measurement of secreted A $\beta$, medium from infected DKO cells carrying APPNL (DKONL) was collected $24 \mathrm{~h}$ after infection. Collected medium was subjected to two-site ELISAs: human/ rat $\beta$-amyloid (40) ELISA Kit (\#294-62501, Wako) and human/rat $\beta$-amyloid (42) ELISA Kit, High Sensitivity (\#292-64501, Wako).

Luciferase assay. Luciferase assay for amyloid precursor protein intracellular domain (AICD) and Notch intracellular domain (NICD) activities was performed as previously described (Imamura et al., 2009; Takagi-Niidome et al., 2013). The \#1210 cell line is a monoclonal DKO cell line stably expressing EGFP, SPC99gvp-6myc, N $\Delta$ E-6myc, UASfirefly luciferase, and TP1-Renilla luciferase genes. AICD and NICD activities were measured by firefly and Renilla luciferases, respectively. Retroviral infection or coinfection of PS1 with point mutations or FLAGPen- 2 was conducted as described above $24 \mathrm{~h}$ after seeding. The cultivation media was renewed $12 \mathrm{~h}$ after virus infection, incubated for another $12 \mathrm{~h}$ at $37^{\circ} \mathrm{C}$. Then the cells were washed with $1 \times \mathrm{PBS}$ once, followed by freeze-thaw cycles 3 times in $-80^{\circ} \mathrm{C}$ for $10 \mathrm{~min}$ and $37^{\circ} \mathrm{C}$ for $20 \mathrm{~min} ; 1 \times$ Picagene Dual Cell Lysis Reagent was added and incubated at room temperature for $2 \mathrm{~h}$. The luminescence was measured by Mithras LB940
(Berthold Technologies) using PicaGeneR Dual SeaPansy Luminescence Kit (Toyo).

In vitro assay. In in vitro assay using crosslinking reagents, $\gamma$-secretase adjusted to $1 \mathrm{mg} / \mathrm{ml}$ from solubilized fraction, was incubated either with $200 \mu \mathrm{M}$ crosslinking reagents or $3 \mathrm{mM} \mathrm{CuSO}_{4}$ and $15 \mathrm{~mm}$ phenanthroline in HEPES buffer [ $10 \mathrm{~mm}$ HEPES, $145 \mathrm{~mm} \mathrm{KCl}, \mathrm{pH} 7.6$ ] containing $0.25 \%$ 3-[(3-cholamidopropyl) dimethylammonio]-2-hydroxypropanesulfonate, $0.05 \%$ phosphatidylcholine, $5 \mathrm{~mm}$ phenanthroline, $10 \mu \mathrm{g} / \mathrm{ml}$ phosphoramidon, and $5 \mathrm{~mm}$ EDTA with complete protease inhibitor mixture for $6 \mathrm{~h}$ at $37^{\circ} \mathrm{C}$. Then $10 \mathrm{~mm} \mathrm{~N}$-ethylmaleimide was added to the buffer to stop the crosslinking reaction. Dissolve $50 \mathrm{~mm}$ E2012 at a final concentration of $100 \mu \mathrm{M}$. Finally, add $150 \mu \mathrm{M}$ purified sC99 $3 \times$ FLAG at a final concentration of $15 \mu \mathrm{M}$, and subsequently incubated the whole solution for $15 \mathrm{~h}$ at $37^{\circ} \mathrm{C}$.

Photoaffinity labeling experiment. Photoaffinity labeling experiment was conducted as described previously (Cai et al., 2017).

Experimental design and statistical analysis. All samples were analyzed in a blind and randomized manner. For quantitative immunoblot analysis, Student's $t$ test was used for comparisons between two-group data, and Tukey's test was used for multiple group comparisons. Statistical analyses were performed by Kyplot or Excel software. 


\section{Results}

\section{SCAM analysis of TMD3 of PS1}

In this study, we constructed mutant PS1 carrying a single cysteine residue from C158 to K187 (single-Cys mt PS1), based on Cys (-) PS1, in which the five endogenous cysteine residues (C92, C158, C263, C410, and C419) are all substituted to serine (Fig. 1) (Sato et al., 2006). We measured secreted $A \beta 40$ and $A \beta 42$ levels in the conditioned medium of Psen $1^{-/-} / P \operatorname{sen} 2^{-/-} \mathrm{DKO}$ cells (Herreman et al., 2000) expressing single-Cys mt PS1, and found that all mutants recovered the $\mathrm{A} \beta$ production (Fig. $2 A$ ). We then tested the water accessibilities of the substituted Cys in these single-Cys mt PS1s by biotinylation experiments using MTSEA-biotin, which requires an aqueous environment to form disulfide bonds with the Cys. With the results that three residues labeled only in the microsome fractions (i.e., C158C, Y159C, and $\mathrm{K} 160 \mathrm{C}$ ) and that labels disappeared from V161C residue, we confirmed that TMD3 spans the lipid bilayer with a Type II orientation, starting from V161 (Fig. 2B, Microsomes). Within the intramembrane region, SCAM analysis of intact cells demonstrated that 10 mutations (i.e., L166C, I167C, L172C, F176C, F177C, F179C, I180C, G183C, E184C, and K187C) reacted with MTSEA-biotin from the extracellular side (Fig. 2B, Intact cells). In addition to these results, SCAM analysis using microsome fractions demonstrated that the PS1 mutants L166C, I167C, and L172C were labeled with MTSEA-biotin. Additionally, biotinylated residues facing the hydrophilic environment align to one side of the helix model, except for I167 (Fig. 2C). To clarify whether the residues face hydrophilic environments inside or outside of the membrane, we next used the charged MTS regents MTSES, MTSET, and MTS-TEAE. MTSES has a small negatively charged group, MTSET has a small positively charged group, and MTS-TEAE has a large positively charged group. These reagents are not able to access closed hydrophilic environments, such as inside the membrane, as labeling competitors in the SCAM analysis (Sato et al., 2008; Takagi et al., 2010; Tominaga et al., 2016; Cai et al., 2017; Cai and Tomita, 2018). In particular, the largest reagent, MTS-TEAE, is thought to be able to only access Cys located in extramembrane regions. Although we did not observe competition at L166C, F176C, F177C, and F179C, the labeling of L172C and I80C was decreased by preincubation with MTSES, and the labeling of G183C, E184C, and K187 was decreased by all MTS reagents (Fig. 3A). These results suggest that L182 is the C-terminal end of TMD3 and that G183 and K187 face the extracellular hydrophilic environment (Fig. 3B).

\section{Topological mapping of TMD3 by crosslinking experiments}

To understand the topological features of the residues that were labeled by MTSEA-biotin in the active $\gamma$-secretase complex, we next conducted crosslinking experiments using PS1 with doubleCys mutations (double-Cys mt PS1) (Tominaga et al., 2016; Cai et al., 2017; Cai and Tomita, 2018). We previously showed that L383 and I387 in TMD7 and L435 in the PAL motif face the catalytic pore (Sato et al., 2006). In this study, we designed several double-Cys mt PS1s, as follows (Fig. 4A): one cysteine was located within TMD3 (L166, L172, F177, I180, or G183), and the other was chosen from the three residues in the CTF (L383, I387, or L435). All these double-Cys mt PS1s retained $\gamma$-secretase activity (Fig. 4B). We also used M2M, M4M, M6M, M8M, M11M, $\mathrm{M} 14 \mathrm{M}$, and M17M, which are sulfhydryl-to-sulfhydryl crosslinking regents with spacer arms of 5.2, 6.5, 7.8, 10.4, 13.0, 16.9, 20.8, and $24.7 \AA$ long, respectively (Loo and Clarke, 2001). Several mutants were crosslinked to the NTF and CTF of PS1 using linkers of varying lengths, except for I180C/L435C (Fig. 5C 4 ), G183C/
A

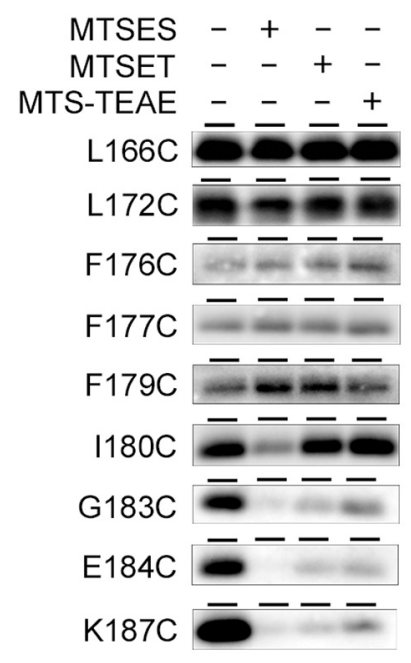

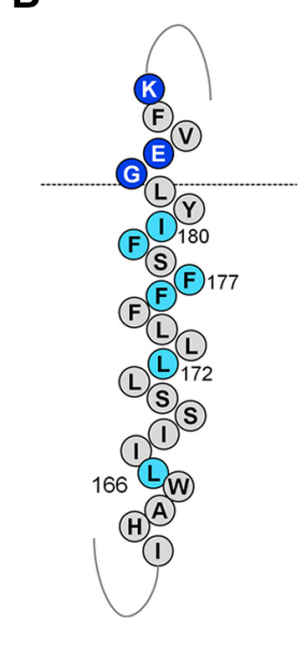

Open hydrophilic environment

Closed hydrophilic environment

Figure 3. SCAM analysis using labeling competitors. $A$, Labeling competition of single-Cys $\mathrm{mt}$ PS1s that were labeled by MTSEA-biotin in Figure $2 B$ was performed after preincubation with negatively charged MTSES, positively charged MTSET, or positively charged bulky MTSTEAE. Immunoblotting assays were performed using the anti-PS1 NTF antibody G1Nr5. B, Summary of the results of SCAM analysis using MTSEA-biotin and competition experiments using charged MTS reagents. Dark blue circles represent the residues that were accessible to all charged reagents (open hydrophilic environment). Light blue circles represent residues that were not competed (closed hydrophilic environment). Gray circles represent residues that were not labeled by MTSEA-biotin.

L383C (Fig. 5A 4 ), G183C/I387C (Fig. 5B $)$, and G183C/L435C (Fig. $5 C_{5}$ ). These results indicated that the hydrophilic interface of TMD3 directly comprises the catalytic pore. Moreover, L172C/L435 (Fig. 5C $_{2}$ ) and F177C/L435C (Fig. 5C $C_{3}$ ) were crosslinked by M8M, whereas L166C/L435C was not (Fig. $5 C_{1}$ ), suggesting that L166 is located farther than the other residues to L435, although these residues are all in the same TMD3. Because L166 is a residue located on the cytosolic side and F177 is on the luminal side of TMD3, the luminal side of TMD3 is inclined in the vertical direction, with the luminal end of TMD3 being furthest from L435 (Figs. $4 A, 5 A_{6}, B_{4}, C_{6}$ ).

\section{Reactivity of substituted Cys in the presence of a transition- state, analog-type $\gamma$-secretase inhibitor}

Next, we conducted the MTSEA-biotin labeling experiments with the preincubation of $\gamma$-secretase with L-685,458. Using this compound as a competitor in the SCAM analysis, we can identify the binding site of L-685,458 around the catalytic site, as well as the allosteric structural changes during the activation of $\gamma$-secretase, because L-685,458 is a substrate-mimicking, transition-state, analog-type $\gamma$-secretase inhibitor that directly binds to catalytic aspartates (Li et al., 2000). As a result, L-685,458 increased the labeling of L166C, L172C, F176C, and F177C, whereas F179C labeling was decreased by this compound (Fig. 6A). These data suggest that the hydrophilic environment around TMD3 is greatly altered during the activation of $\gamma$-secretase, particularly around $\mathrm{F} 177$. We then analyzed the effect of L-685,458 on the crosslinking of F177C/I387C double-Cys mt PS1. We previously demonstrated that the labeling of I387C was unaffected by L-685,458 (Sato et al., 2006), and the water accessibility of F177C was increased by sixfold. However, the efficiency of crosslinking of the double-Cys mt PS1 was significantly reduced, 
A

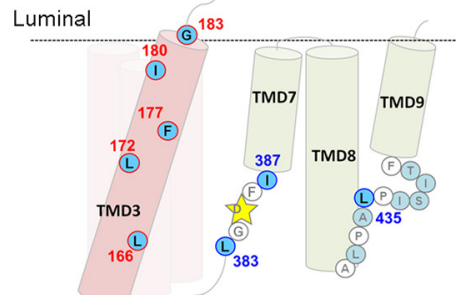

Cytosolic
B

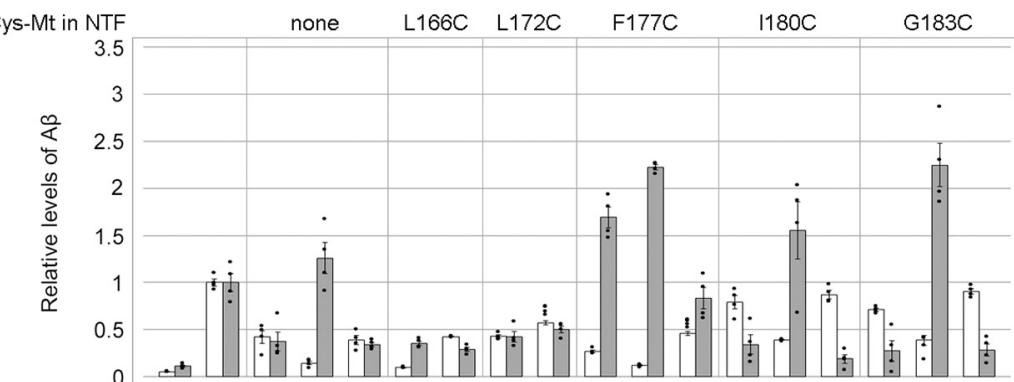

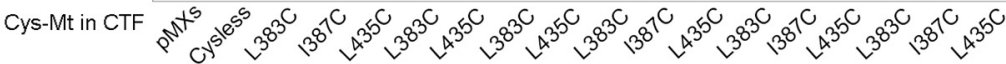

Figure 4. $\quad \gamma$-Secretase activity of single- or double-Cys mt PS1s used in topology mapping. A, Location of the Cys mutations analyzed in this study. Blue represents Cys mutations. Yellow star indicates the catalytic aspartate. $B$, Sandwich ELISA of secreted A $\beta$ from APPNL-stable DKO cells transiently transfected with single- or double-Cys mt PS1 ( $n=4$, mean \pm SEM). The levels of secreted $A \beta$ in the conditioned media were normalized by that of cells expressing PS1/Cys (-). White bars represent production of $A \beta 40$. Gray bars represent production of $A \beta 42$.
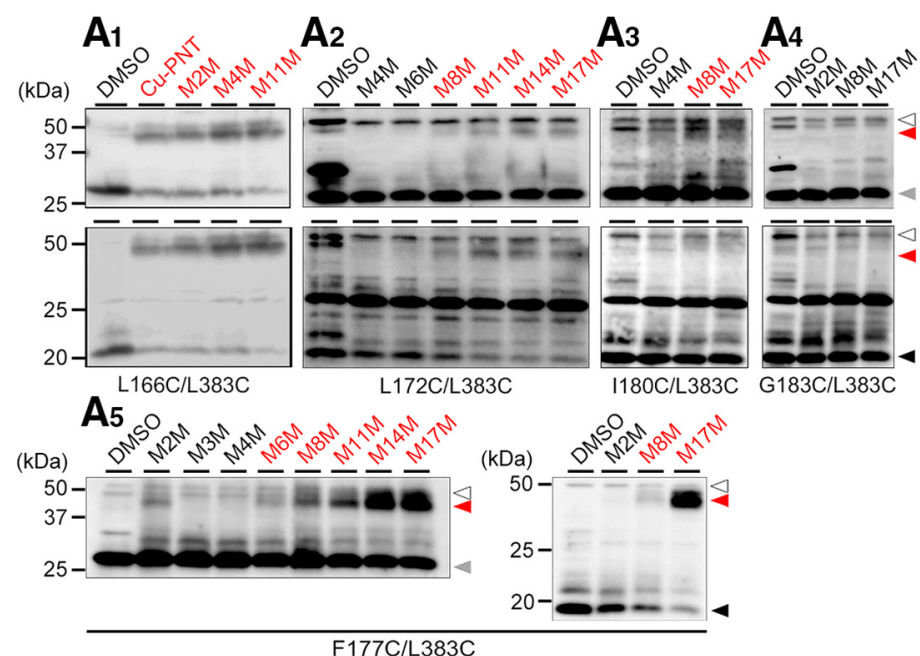

$B_{1}$

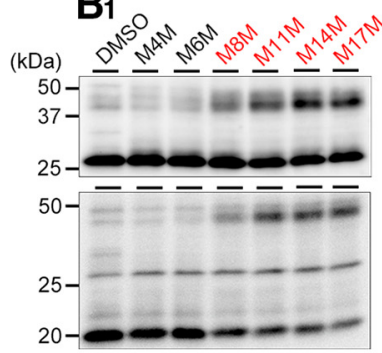

$\mathrm{F} 177 \mathrm{C} / 1387 \mathrm{C}$
$\mathrm{B}_{2}$

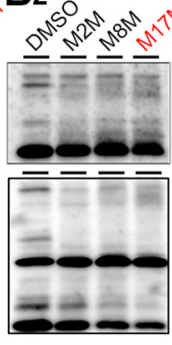

$1180 \mathrm{C} / 1387 \mathrm{C}$
$\mathrm{B}_{3}$

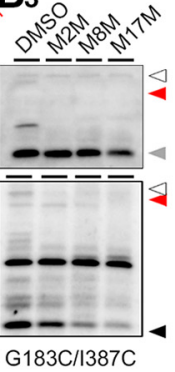

A6

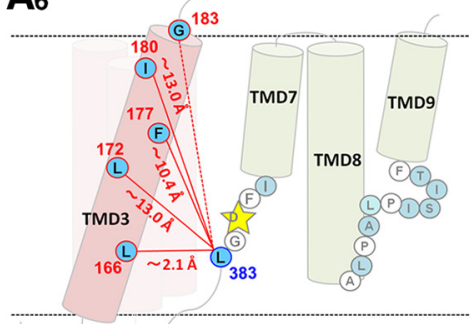

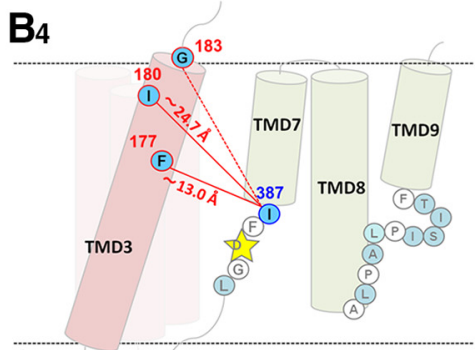

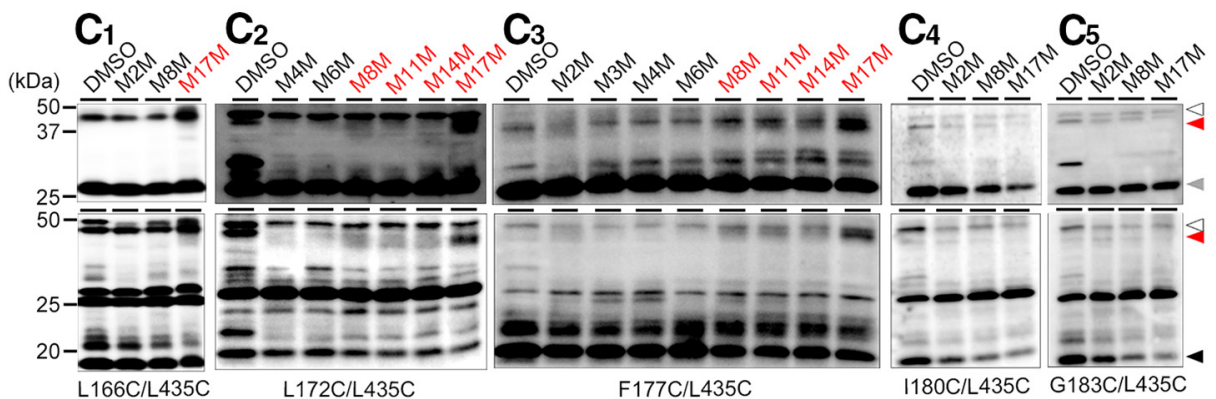

$\mathbf{C}_{6}$

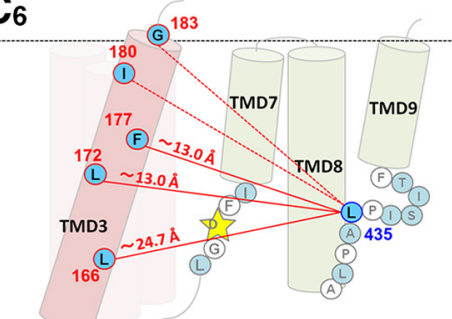

Figure 5. Crosslinking experiments using catalysts or MTS crosslinkers. Crosslinking experiments of double-Cys mt PS1 with Cys mutations in TMD3 and L383C ( $\boldsymbol{A})$, I387C ( $\boldsymbol{B})$, or L435C ( $\boldsymbol{C}$ ). Immunoblotting assays were performed using the anti-PS1 NTF antibody G1Nr5 and anti-PS1CTF antibody G1L3. Gray and black arrowheads represent S1 NTFs and PS1 CTFs, respectively. White and red arrowheads represent full-length PS1 and crosslinked products, respectively. Red represents crosslinkers that link the two Cys in double-Cys mt PS1. Right, Red solid lines indicate the predicted maximum lengths between two residues. Dotted lines indicate binding two residues where crosslink could not be detected. Light blue circles represent positions of the crosslinked Cys. Yellow stars indicate the catalytic aspartates. 
A

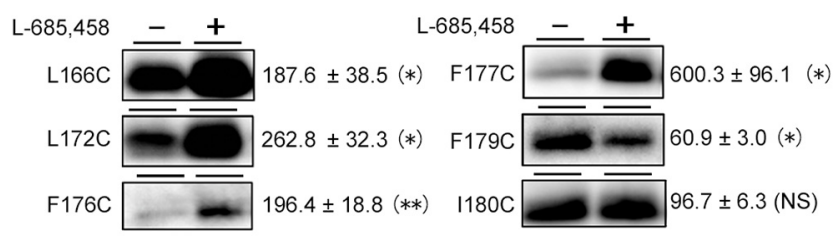

B

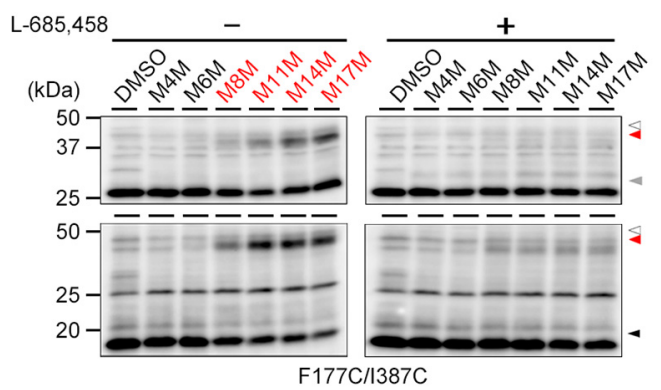

Figure 6. Labeling competition by L-685,458. A, Labeling of single-Cys mt PS1 by MTSEAbiotin was performed after preincubation with $L-685,458$. Immunoblotting assays were performed using an anti-PS1 NTF antibody. Labeling efficiencies of biotinylated proteins are shown to the right of each panel $\left(n=3\right.$; mean \pm SEM). ${ }^{*} p<0.05$ (Student's paired $t$ test). ${ }^{* *} p<$ 0.01 (Student's paired $t$ test). $\boldsymbol{B}$, Crosslinking experiments of F177C/I387C double-Cys mt PS1 with L-685,458. Immunoblotting assays were performed using the anti-PS1 NTF antibody G1Nr5 and anti-PS1 CTF antibody G1L3. Gray and black arrowheads represent PS1 NTFs and PS1 CTFs, respectively. White and red arrowheads represent full-length PS1 and crosslinked products, respectively. Red represents crosslinkers that link the two Cys in the double-Cys mt PS1. NS, not significant.

suggesting that the distance between F177C and I387C was extended by L-685,458 (Fig. 6B). Collectively, these data indicated that dynamic conformational changes occur in TMD3 during the activation of PS1.

\section{Conformational changes in TMD3 are associated with changes in $\mathrm{A} \boldsymbol{\beta} 42$ production}

We previously analyzed the molecular mechanism of $\gamma$-secretase modulators (GSMs) that decrease A $\beta 42$ generation using photoaffinity labeling and SCAM. These compounds target either TMD1 and HL1 of PS1 to induce large conformational changes to activate PS1 trimming activity, thereby reducing $\mathrm{A} \beta 42$ production (Ohki et al., 2011; Takeo et al., 2014; Cai et al., 2017). However, the effect of conformational changes in TMD3 on A $\beta 42$ production remains unclear. Notably, a number of FAD mutations have been identified in TMD3 so far, suggesting that TMD3 is a crucial domain for the regulation of $\mathrm{A} \beta 42$ production.

To investigate the correlation of the water accessibility of TMD3- and A $\beta 42$-generating activity, first we analyzed the effect of 4 FAD-linked A $\beta 42$-increasing mutations (i.e., P117L at HL1, M139V at TMD2, F177L at TMD3, E184D at HL3) (Yasuda et al., 1997; Wisniewski et al., 1998; Finckh et al., 2000; Rogaeva et al., 2001) on the MTSEA-biotin labeling of L166C and F177C. In addition to $A \beta 42$ ratio, pathological effects of these mutations were assessed by measuring the production of AICD and NICD, which are the intracellular counterpart product of the $\gamma$-secretasemediated proteolysis. All these FAD mutations increased the A $\beta 42$ production ratio (Fig. $7 A_{1}, A_{2}$ ) and decreased the production of both AICD and NICD (Fig. $7 B$ ). Intriguingly, FAD mutations reduced the labeling efficiencies at either L166C or F177C (Fig. 7C,D), suggesting that these mutations induced that conformational changes in TMD3.

We then analyzed the effect of the elongation of $\mathrm{N}$-terminal end of Pen-2, which leads to the $\mathrm{A} \beta 42$ overproduction (Fig. $7 A_{3}$ )
(Isoo et al., 2007). However, the elongated Pen-2 affected neither the AICD/NICD production nor the hydrophilicity around L166C/F177C (Fig. 7 B,E). We previously showed that the elongated Pen-2 decreased the water accessibility of the catalytic pore (Isoo et al., 2007). Thus, these results suggest that Pen-2 affects the $\mathrm{A} \beta 42$ ratio by direct effect on the conformation of the catalytic center.

Next, we analyzed the effect of a genetic PS1 mutation that reduces $\mathrm{A} \beta 42$ production. In systematic mutagenesis for SCAM, we noticed that the V236C mutation in Cys (-) PS1 at TMD5 significantly reduced the $\mathrm{A} \beta 42$ production ratio (Tominaga et al., 2016). Then we identified the V236S substitution of PS1 as an A 342 -reducing mutation (Fig. $7 A_{4}$ ). Notably, the V236S mutation significantly increased the labeling of both L166C and F177C (Fig. 7C), suggesting that $\mathrm{A} \beta 42$-generating activity is inversely correlated with the water accessibility of L166 and F177. Supporting this notion, SCAM analysis using intact cells preincubated with A $\beta 42$-reducing GSM (i.e., ST1120) showed increased labeling of both L166C and F177C (Fig. 7F). These results together suggest that the water accessibility of TMD3 in the catalytic pore is increased when the trimming activity of the enzyme is enhanced by genetic mutations or GSM.

To clarify the conformation changes that trigger the enlargement of the hydrophilic environment around TMD3, we next performed crosslinking experiments using L166C/L383C and F177C/I387C double-Cys PS1 mutants with the P117L mutation, to measure the lengths between these two designated Cys when the $\mathrm{A} \beta 42$ production ratio is altered. However, the $\mathrm{P} 117 \mathrm{~L}$ mutation did not alter the crosslinking patterns either of L166C/L383C or F177C/I387C (Fig. 8). These double-Cys PS1 mutants were also preincubated with E2012, before conducting the crosslinking experiment. E2012 is another phenylimidazole-type GSM, which has the same basic structure as ST1120 (Pozdnyakov et al., 2013; Cai et al., 2017). We confirmed that the two double-Cys mutants retained their response toward E2012 (Fig. 9A). However, preincubation of E2012 at an A $\beta 42$-lowering concentration did not change the crosslinking of either L166C/L383C or F177C/I387C (Fig. $9 B$ ). These results together demonstrate that changes in the length between TMD3 and TMD7 do not just simply lead to the regulation of $\mathrm{A} \beta 42$ production.

Finally, to test whether conformational changes in TMD3 are prerequisites for the increased trimming of $\mathrm{A} \beta$, we analyzed $\mathrm{A} \beta$ production from double-Cys mt PS1, in which TMD3 and TMD7 were stabilized. Crosslinking experiments using solubilized membrane fractions from DKO cells expressing the L166C/ L383C and F177C/I387C mutants demonstrated that these residues are crosslinked by $\mathrm{Cu}-\mathrm{PNT}$ and the M8M crosslinker, respectively. $\mathrm{Cu}-\mathrm{PNT}$ acts as a redox catalyst to form a disulfide bond in Cys that can collide (Klco et al., 2003). The solubilized fraction showed $\gamma$-secretase activity in vitro, which was sensitive to incubation with E2012. Notably, the membrane fraction of the L166C/L383C double-Cys PS1 mutant that was precrosslinked with $\mathrm{Cu}-\mathrm{PNT}$ lost its sensitivity against E2012, whereas its $\mathrm{A} \beta 42$ production ratio was comparable with that without crosslinking (Fig. 10A). In addition, a photoaffinity labeling experiment confirmed that crosslinked L166C/L383C retained the same affinity toward E2012 (Fig. 10B). Together, the conformational changes in TMD3 are crucial for the regulation of $\mathrm{A} \beta 42$ production.

\section{Discussion}

In this study, we found that several amino acid residues in TMD3 face the hydrophilic catalytic pore in the lipid bilayer, and a conformational change in TMD3 is a prerequisite for the activation 

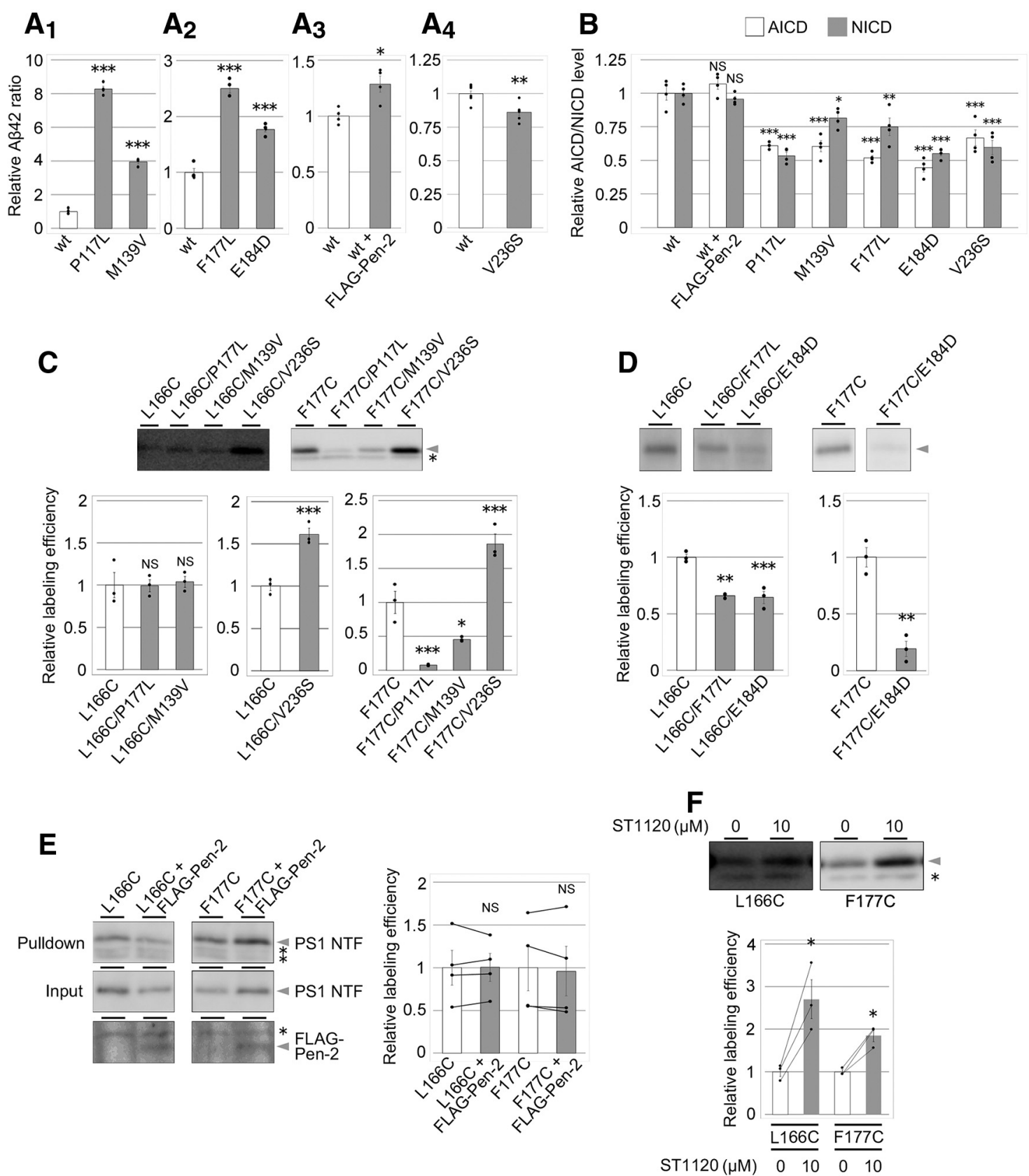

Figure 7. Alteration of the hydrophilic environment around TMD3 is associated with A 342 production. $A$, Conditioned media of DKONL cells transiently transfected with PS1 carrying a point mutation or cotransfected with FLAG-Pen-2 were analyzed by sandwich ELISA. A $\beta 42$ ratios [percentage of A $\beta 42 /(A \beta 40+A \beta 42)$ ] were normalized by that of cells expressing PS1wt $\left(\boldsymbol{A}_{7}, A_{2}: n=\right.$ 4 ; mean \pm SEM; ${ }^{* * *} p<0.001$, compared with control by Tukey's test; $A_{3}: n=4$; mean $\pm S E M ;{ }^{*} p<0.05$, compared with control by Student's $t$ test; $A_{4}: n=6$; mean \pm SEM; ${ }^{* *} p<0.01$, compared with control by Student's $t$ test). $\boldsymbol{B}$, AICD and NICD production by PS1 mutant or PS1/FLAG-Pen-2 was measured by luciferase assay. Measured fluorescence was normalized by the expressing level of each PS1; then data were normalized by that of wt $\left(n=4\right.$; mean \pm SEM). ${ }^{*} p<0.05$, compared with control (Tukey's test). ${ }^{* *} p<0.01$, compared with control (Tukey's test). ${ }^{* * *} p<0.001$, compared with control (Tukey's test). C, SCAM analysis of L $166 C$ or F177C single-Cys mt PS1 with a point mutation by MTSEA-biotin. Immunoblotting assays were performed using the anti-PS1 NTF antibody G1Nr5. Gray arrowhead and asterisk represent PS1 NTF and a nonspecific protein, respectively. Quantifications of the relative labeling efficiency of each PS1 are shown below $\left(n=3\right.$; mean \pm SEM). ${ }^{*} p<0.05$, compared with control (Tukey's test). ${ }^{* * *} p<0.001$, compared with control (Tukey's test). D, SCAM analysis of L166C or F177C single-Cys mt PS1 with a point mutation in TMD3 by MTSEA-biotin. Immunoblotting assays were performed using the anti-PS1 NTF antibody G1Nr5. Gray arrowhead represents PS1 NTF. Quantifications of the relative labeling efficiency of each PS1 are shown below $\left(n=3\right.$; mean \pm SEM). ${ }^{* *} p<0.01$, compared with control by Tukey's test for L166C $\mathrm{mt}$ and Student's $t$ test for F177C $\mathrm{mt}$. ${ }^{* * *} p<0.001$, compared with control by Tukey's test for L166C $\mathrm{mt}$ and Student's $t$ test for F177C mt. $\boldsymbol{E}$, SCAM analysis of L166C or F177C single-Cys mt PS1 FLAG-Pen-2 by MTSEA-biotin. Immunoblotting assays were performed using the anti-PS1 NTF antibody G1Nr5 and anti-FLAG-tag antibody anti-FLAG M2. Gray arrowhead and asterisk represent the target proteins and a nonspecific protein, respectively. Quantifications of the relative labeling efficiency of each PS1 are shown at the right side ( $n=4$; mean \pm SEM; not significant, compared with control by paired Student's $t$ test). $F$, SCAM analyses of L166C and F177C single-Cys mt PS1 by MTSEA-biotin were performed after preincubation with ST1120. Immunoblotting assays were performed using the anti-PS1 NTF antibody G1Nr5. Gray arrowhead and asterisk represent PS1 NTF and a nonspecific protein, respectively. Quantifications of the relative labeling efficiency of each PS1 are shown below $\left(n=3 ;\right.$ mean \pm SEM). ${ }^{*} p<0.05$, compared with DMSO (paired Student's $t$ test). NS, not significant. 

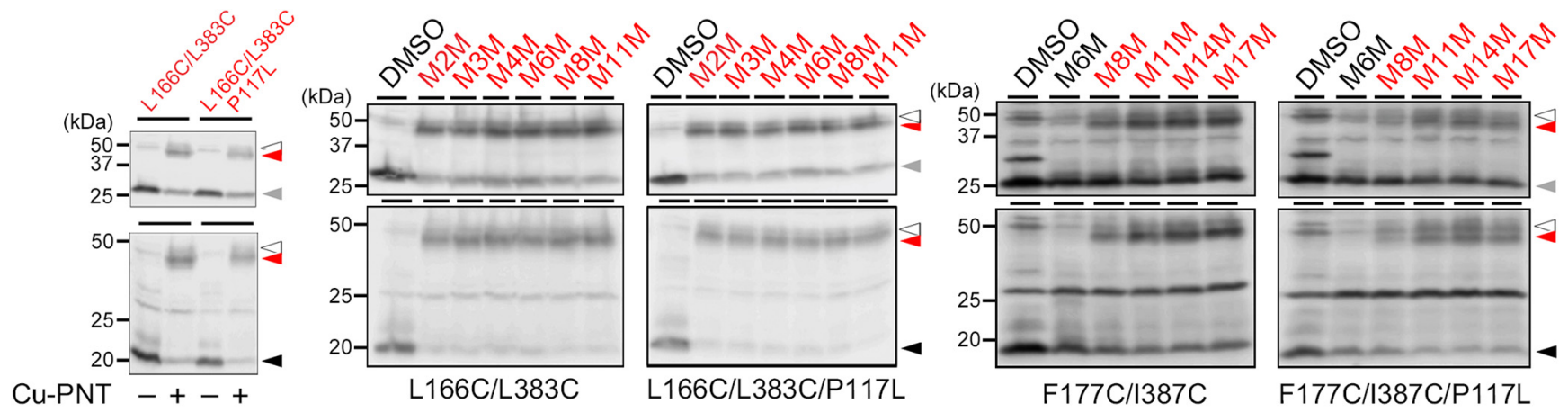

Figure 8. No effect of FAD mutations on the crosslinking between TMD3 and TMD7. Crosslinking experiments of L166C/L383C and F177C/I387C double-Cys PS1 mutants with FAD mutations. Immunoblotting assays were performed using the anti-PS1 NTF antibody G1Nr5 and anti-PS1 CTF antibody G1L3. Gray and black arrowheads represent PS1 NTFs and PS1 CTFs, respectively. White and red arrowheads represent full-length PS1 and crosslinked products, respectively. Red represents crosslinkers that shifted the bands.

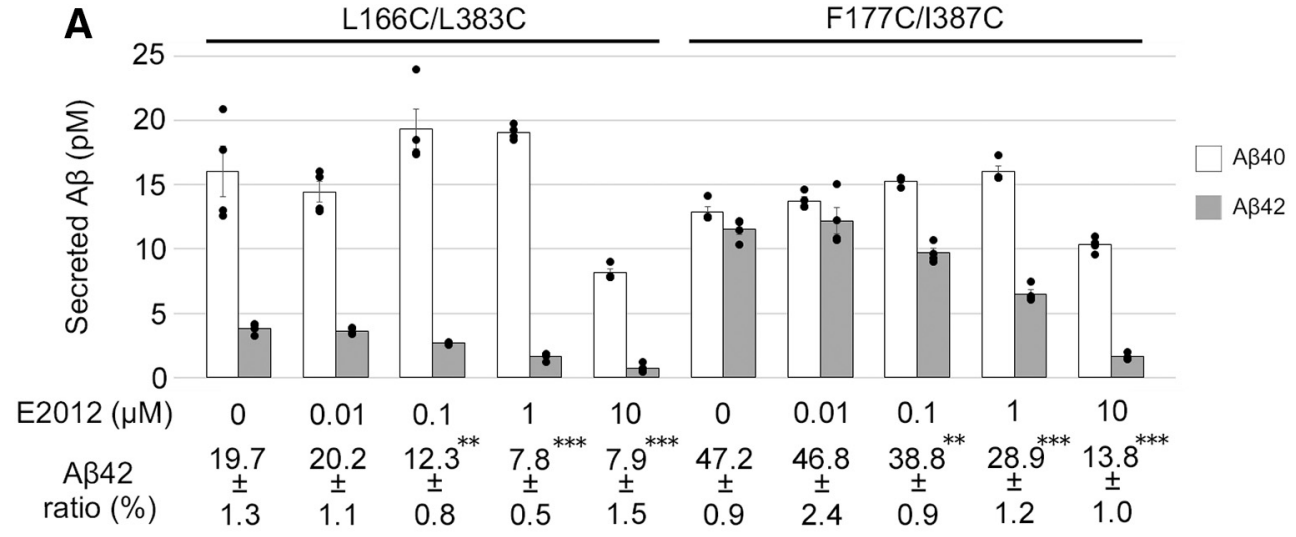

\section{B}
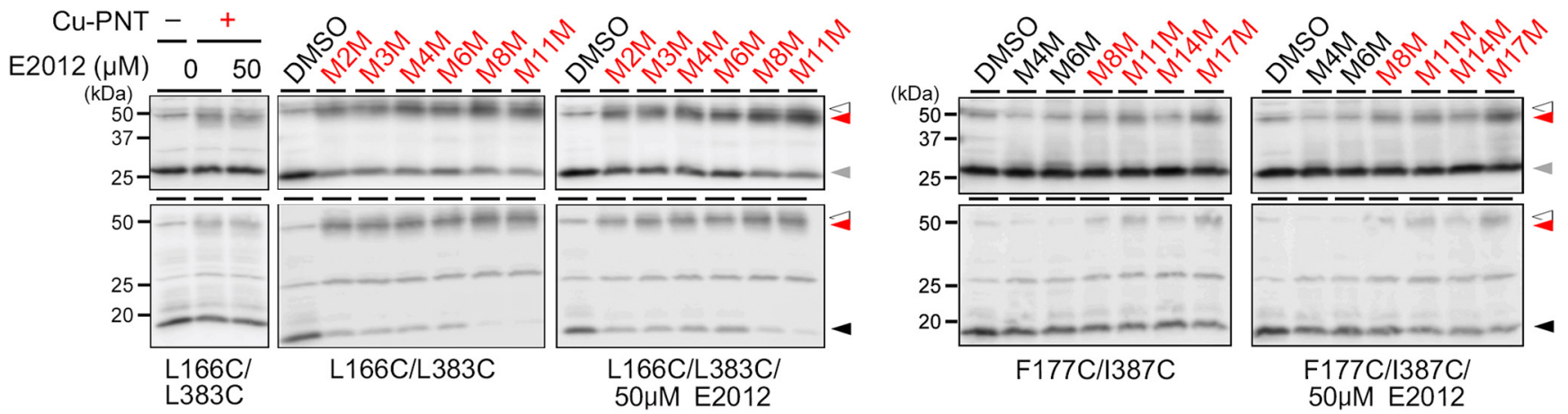

Figure 9. No effect of $\gamma$-secretase modulators on the crosslinking between TMD3 and TMD7. A, Conditioned media of DKONL cells expressing L166C/L383C or F177C/1387C double-Cys PS1 mutants with incubation of $E 2012$ were analyzed by sandwich ELISA. $A \beta 42$ ratios [percentage of $A \beta 42 /(A \beta 40+A \beta 42)$ ] are shown below the graph $\left(n=4\right.$; mean \pm SEM). ${ }^{* *} p<0.01$, compared with DMSO (Tukey's test). ${ }^{* * *} p<0.001$, compared with DMSO (Tukey's test). B, Crosslinking experiments of L166C/L383C and F177C/I387C double-Cys PS1 mutants with preincubation with $50 \mu \mathrm{M}$ E2012. Immunoblotting assays were performed using the anti-PS1 NTF antibody G1Nr5 and anti-PS1 CTF antibody G1L3. Gray and black arrowheads represent PS1 NTFs and PS1 CTFs, respectively. White and red arrowheads represent full-length PS1 and crosslinked products, respectively. Red represents crosslinkers that shifted the bands.

of the trimming activity of $\gamma$-secretase. We previously found that swapping TMD3 to an unrelated TMD failed to abolish $\gamma$-secretase activity but caused significant overproduction of A $\beta 42$ (Watanabe et al., 2005). Previous investigation using alanine scanning revealed that several alanine substitutions in the TMD3 region affect the potency of some kinds of $\gamma$-secretase inhibitor (Zhao et al., 2008). Moreover, $\sim 30$ FAD mutations, including L166P, which is one of the strongest FAD mutations ever found (Moehlmann et al., 2002), have been identified in TMD3, which account for $>10 \%$ of all the 219 FAD mutations found in PS1. This is a large number considering that TMD3 consists of only $\sim 20$ residues, which account for $<5 \%$ of the entire sequence. In addition, FAD mutations in TMD3 can attenuate the sensitivity toward $\gamma$-secretase inhibitors (Kornilova et al., 2006; Page et al., 2008). Together, it is plausible that TMD3 plays an important role in the regulation of $\mathrm{A} \beta 42$ production ratio.

Recently, the atomic structure of PS1 in human $\gamma$-secretase has been clarified by cryo-EM single-particle analysis (Bai et al., 2015). The authors analyzed 400,000 $\gamma$-secretase molecules and divided them into several groups containing the three major conformers according to their structural characteristics. In this study, distances between TMD3 and the other residues were measured by crosslinking experiments. First, G183 was unable to 
A

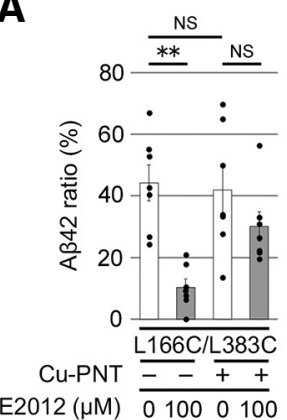

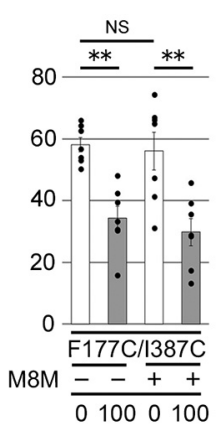

$\mathbf{B}$

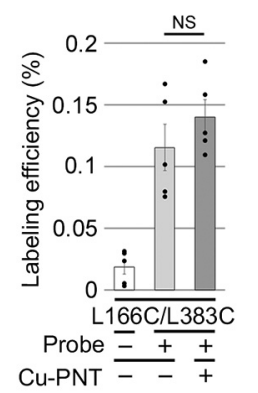

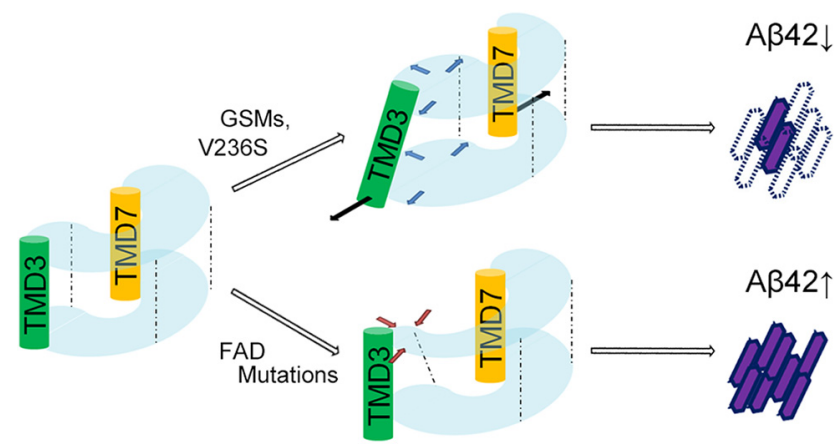

Figure 10. Conformational dynamics around L166 is crucial for the effect of $\gamma$-secretase modulators. $A$, In vitro $A \beta 42$ production ratios [percentage of $A \beta 42 /(A \beta 40+A \beta 42)$ ] were measured by sandwich ELISA $\left(n=7\right.$; mean \pm SEM). ${ }^{* *} p<0.01$ (Tukey's test). $\boldsymbol{B}$, Photoaffinity labeling experiment demonstrated that crosslinked L166C/L383C still retains the same level of affinity toward E2012 ( $n=5$; mean \pm SEM; not significant by Student's $t$ test). Immunoblotting assays were performed using the anti-PS1 NTF antibody G1Nr5. Cu-PNT were incubated at a final concentration of 3 and $15 \mathrm{~mm}$, respectively. Probe compounds were added at a final concentration of $20 \mu \mathrm{M}$. NS, not significant.

crosslink with L383, I387, and L435 (Fig. $5 A_{4}, B_{3}, C_{5}$ ), even with the longest crosslinker ( $24.7 \AA$ length), whereas the distance between those residues in the atomic structure clarified by cryo-EM was $21 \AA$. This might be due to steric hindrance by TMD5 and/or TMD7 located between G183 and L383, I387, or L435 in all the three major conformers. Next, distances between L172 or F177 and L435 (Fig. $5 C_{2}, C_{3} ; \sim 13$ and $10 \AA$, respectively) identified by the crosslinking experiment were shorter than those measured from the atomic structure (22-28 $\AA$ ). L435 is a residue in the PAL motif, which forms the catalytic pore and is important for $\gamma$-secretase activity (Tomita et al., 2001; Sato et al., 2008). Thus, these discrepancies might be caused by the flexibility of the conformation of the catalytic pore. In addition, L166 and L383 could be linked directly by disulfide bonding using Cu-PNT crosslinking (Fig. $5 A_{1}$ ), although the distance was $10-18 \AA$ based on the atomic structure. However, positions of these two residues were highly variable among the three major conformers of PS1 identified by cryo-EM, supporting our notion that dynamic structural changes occur in TMD3 and TMD7 along with activation of the enzyme. Thus, the conformation of TMD3 around L166 is highly flexible and plays a crucial role in $\gamma$-secretase-mediated intramembrane proteolysis.

Eight residues within TMD3 were strongly labeled in the SCAM experiments (Fig. 2B). Among them, I167 might be facing a unique hydrophilic environment because I167C was the only residue to be labeled in the microsome fraction, and all residues other than I167 align on one side in the helix model (Fig. 2C). In the competition SCAM assay using L-685,458, the labeling of L166C, L172C, F176C, and F177C was increased (Fig. 6A). L-685,458 is a transition-state, analog-type $\gamma$-secretase inhibitor that directly binds to catalytic aspartates (Shearman et al., 2000; Evin et al., 2005). When L-685,458 binds to the catalytic site, PS1 is expected to take the active conformation. Thus, the increased labeling of several TMD3 residues by $\mathrm{L}-685,458$ is thought to reflect enlargement of the catalytic pore because the labeled Cys in TMD3 (i.e., L166C, L172C, and F177C) were all crosslinked with both L383 and I387 (Fig. $5 A_{1}, A_{2}, A_{5}, B_{1}$ ), which face the pore (Sato et al., 2006). However, most of these residues face the outside of PS1 in the cryo-EM structure. Therefore, these results suggest that TMD3 rotates toward the catalytic site in the active conformation.

We found a close inverse correlation between changes in the hydrophilic environment around TMD3 and $\mathrm{A} \beta 42$ production

Figure 11. Association between alterations in the production of $A \beta 42$ and changes in the hydrophilic environment around TMD3. Summary of the results of SCAM analyses and crosslinking experiments. Changes in the $A \beta 42$ ratio are closely associated with alterations in the hydrophilic environment around TMD3. When the $A \beta 42$ ratio is decreased, the hydrophilic environment around TMD3 is enlarged (top). In particular, changes in the cytosolic side are regulated by TMD3 and TMD7. On the other hand, when the $A \beta 42$ ratio is increased, the hydrophilic environment around F177 is narrowed, whereas no changes can be observed around L166 (bottom).

ratios. An increase and decrease in $\mathrm{A} \beta 42$ ratios by genetic mutations or GSM caused a decrease and increase, respectively, of L166C and F177C labeling (Figs. 7C,D, 11). In the atomic structure, TMD3 is largely inclined in the vertical direction, with the cytosolic side becoming farthest from the catalytic site (Fig. 4A). We found that the all three FAD mutations (P117L, M139V, and E184D) decreased the labeling of F177 (Fig. 7C,D). However, the results of our crosslinking experiments showed slightly different results, with no changes in the crosslinking patterns of the F177C/ I387C double-Cys mutant (Figs. 8, 9B). The hydrophilic catalytic pore is formed by several TMDs, including TMD3, whereas the crosslinking experiment measures the distance between two specific domains. Thus, it is possible that alteration of the hydrophilic environment around F177 is not mediated by the spatial distance between TMD3 and TMD7. Indeed, we previously found that a number of residues in TMD1, TMD4, and TMD5 face the catalytic pore (Takagi et al., 2010; Tominaga et al., 2016). The atomic structure of these regions shows that the sequence between M233 and I238 in TMD5 is spatially located near F177. Furthermore, several FAD mutations (Campion et al., 1999; Sodeyama et al., 2001; Park et al., 2008; Ting et al., 2014) and an A $\beta 42$-decreasing mutation (Fig. $7 A_{4}$ ) have been identified in all, except for A234 and V236. Together, this area in TMD5 appears to be crucial for regulating $A \beta 42$ production and is potentially able to affect the hydrophilic environment around F177.

We also clarified that GSMs do not have any effect on the results of the crosslinking experiments (Fig. 9B). However, in vitro analysis showed that the effect of E2012 was attenuated by crosslinking catalysts when L166C, which is located on the cytosolic side of TMD3, and L383C are linked and stabilized (Fig. $10 A$ ). The drawback of crosslinking experiments is that the reagents link two Cys in the closest conformation. If two Cys are spatially lengthened with no change in the closest distance, the crosslinking reagents cannot distinguish the difference. Thus, we still believe that elongation of TMD3 and TMD7, particularly on the cytosolic side, is crucial for $A \beta 42$ downregulation. Several previous studies have shown that GSM caused an open conformation of PS1, whereas FAD mutation induced the narrow and closed conformation by fluorescence lifetime imaging microscopic analyses (Lleó et al., 2004; Berezovska et al., 2005; Uemura et al., 2009). Recent analysis also revealed that $\gamma$-secretase inhibitor leads to more population of closed conformation (Elad et al., 
2015). Moreover, the cytosolic side of TMD3 makes a dynamic movement between closed and opened conformations in the $\gamma$-secretase simulation models (Somavarapu and Kepp, 2017). Our findings are highly consistent with these reports, providing a new insight that PS1 in activated $\gamma$-secretase takes an open conformation with the cytosolic side of TMD3 being far from the catalytic center. Indeed, we also previously demonstrated that the mobility on the cytosolic side of TMD4 affects the $\mathrm{A} \beta$ production ratio (Tominaga et al., 2016), suggesting that the structural changes in TMD3 and TMD4 are correlated and affect mutually.

Although the functional roles of the conformation changes of TMD3 are still unclear, recent reports raise some interesting possibilities. The first insight comes from Escherichia coli rhomboid GlpG, which is also an intramembrane protease containing a catalytic cavity inside the complex. The combination of the $\mathrm{x}$-ray crystal structure and molecular dynamics simulation suggests that four residues (one histidine, two serines, and one glutamine) retain water molecules by hydrogen bonds and are positioned deeply inside the catalytic cavity when the gate is opened to recruit the substrate, so that the catalytic serine can hydrolyze the substrate (Zhou et al., 2012; Strisovsky, 2013). Interestingly, one histidine (H163) and two serines (S169 and S170) can be found in the cytosolic side of TMD3, whereas few glutamines are located in the cytosolic side of TMD5. Although further work is needed to clarify the role of these residues in PS1, considering that the movement of L166 is crucial for A $\beta 42$ regulation and that L166 is directly linked with L383, the L166 region can be considered to be the key toward determining the cleavage efficiency mediated by $\gamma$-secretase. We speculate that these "water-retaining residues" hold water molecules and pass them on to the catalytic site simultaneously with conformational changes in TMD3 that are associated with $\gamma$-secretase activation. Another possible function of TMD3 is destabilization of the helical conformation of the substrate. L166, which shows a high water accessibility in TMD3 (Fig. $2 B$ ), is located spatially close to T714 and V715 of substrate in the cryo-EM structure (Zhou et al., 2019). These residues are the critical residues in the determination of the trimming activity of the $\gamma$-secretase $(\mathrm{Xu}, 2009)$. Furthermore, $\alpha$-helix is known to be prone to be destabilized under hydrophilic environment (Soman et al., 1991). Thus, we speculate the TMD3-facing hydrophilic environment, especially around L166, is the key to unwind the helix structure to promote the trimming of the $\gamma$-secretase.

In conclusion, using biochemical structural analysis, we demonstrated that TMD3 forms a catalytic pore, and the hydrophilic environment around the domain changes significantly along with alterations in $A \beta 42$ production. A recent study revealed that W165 and S169 in TMD3 interact with V715 and I712 in APP, respectively, before the initial $\varepsilon$-cleavage occurs (Zhou et al., 2019). Moreover, A713 is the bending residue of APP and provides high flexibility to the following helix (Itkin et al., 2017). Considering our finding that the topological changes between the $\mathrm{N}$-terminal side of TMD3 from the catalytic center are correlated with $\mathrm{A} \beta$ production, we hypothesize TMD3 as an optimizing domain of the substrate position during the $\gamma$-cleavage. And conformational changes in TMD3 and TMD7 might be involved in the induction of hybrid $\beta$-sheet structure formed by PS1 TMD6 and the substrate (Zhou et al., 2019). Structural analysis using DAPT, which prevents the trimming activity (Zhao et al., 2004), would shed light on the importance of TMD3 during the $\gamma$-cleavage. Nevertheless, further integrated analysis (i.e., molecular dynamics simulation with lipid, water molecules, and GSMs), in addition to conventional structural analysis of PS, will provide crucial information for the further understanding the precise roles of TMD3 in regulating $\mathrm{A} \beta$ production.

\section{References}

Bai XC, Rajendra E, Yang G, Shi Y, Scheres SH (2015) Sampling the conformational space of the catalytic subunit of human $\gamma$-secretase. Elife 4:e11182.

Berezovska O, Lleo A, Herl LD, Frosch MP, Stern EA, Bacskai BJ, Hyman BT (2005) Familial Alzheimer's disease presenilin 1 mutations cause alterations in the conformation of presenilin and interactions with amyloid precursor protein. J Neurosci 25:3009-3017.

Cai T, Tomita T (2018) Structural analysis of target protein by substituted cysteine accessibility method. Bio-Protocol 8:1-11.

Cai T, Yonaga M, Tomita T (2017) Activation of $\gamma$-secretase trimming activity by topological changes of transmembrane domain 1 of presenilin 1 . J Neurosci 37:12272-12280.

Campion D, Dumanchin C, Hannequin D, Dubois B, Belliard S, Puel M, Thomas-Anterion C, Michon A, Martin C, Charbonnier F, Raux G, Camuzat A, Penet C, Mesnage V, Martinez M, Clerget-Darpoux F, Brice A, Frebourg T (1999) Early-onset autosomal dominant Alzheimer disease: prevalence, genetic heterogeneity, and mutation spectrum. Am J Hum Genet 65:664-670.

Elad N, De Strooper B, Lismont S, Hagen W, Veugelen S, Arimon M, Horré K, Berezovska O, Sachse C, Chavez-Gutierrez L (2015) The dynamic conformational landscape of $\gamma$-secretase. J Cell Sci 128:589-598.

Evin G, Canterford LD, Hoke DE, Sharples RA, Culvenor JG, Masters CL (2005) Transition-state analogue $\gamma$-secretase inhibitors stabilize a 900 $\mathrm{kDa}$ presenilin/nicastrin complex. Biochemistry 44:4332-4341.

Finckh U, Müller-Thomsen T, Mann U, Eggers C, Marksteiner J, Meins W, Binetti G, Alberici A, Hock C, Nitsch RM, Gal A (2000) High prevalence of pathogenic mutations in patients with early-onset dementia detected by sequence analyses of four different genes. Am J Hum Genet 66: $110-117$.

Herreman A, Serneels L, Annaert W, Collen D, Schoonjans L, De Strooper B (2000) Total inactivation of $\gamma$-secretase activity in presenilin-deficient embryonic stem cells. Nat Cell Biol 2:461-462.

Imai S, Cai T, Yoshida C, Tomita T, Futai E (2018) Specific mutations in presenilin 1 cause conformational changes in $\gamma$-secretase to modulate amyloid $\beta$ trimming. J Biochem 165:37-46.

Imamura $\mathrm{Y}$, Watanabe N, Umezawa N, Iwatsubo T, Kato N, Tomita T, Higuchi $\mathrm{T}$ (2009) Inhibition of gamma-secretase activity by helical betapeptide foldamers. J Am Chem Soc 131:7353-7359.

Isoo N, Sato C, Miyashita H, Shinohara M, Takasugi N, Morohashi Y, Tsuji S, Tomita $T$, Iwatsubo $T$ (2007) A $\beta 42$ overproduction associated with structural changes in the catalytic pore of $\gamma$-secretase: common effects of Pen-2 N-terminal elongation and fenofibrate. J Biol Chem 282: $12388-12396$.

Itkin A, Salnikov ES, Aisenbrey C, Raya J, Glattard E, Raussens V, Ruysschaert J, Bechinger B (2017) Structural characterization of the amyloid precursor protein transmembrane domain and its $\gamma$-cleavage site. ACS Omega 2:6525-6534.

Iwatsubo T, Odaka A, Suzuki N, Mizusawa H, Nukina N, Ihara Y (1994) Visualization of $A \beta 42(43)$ and $A \beta 40$ in senile plaques with end-specific $\mathrm{A} \beta$ monoclonals: evidence that an initially deposited species is $\mathrm{A} \beta 42(43)$. Neuron 13:45-53.

Karlin A, Akabas MH (1998) Substituted-cysteine accessibility method. Methods Enzymol 293:123-145.

Kitamura T, Koshino Y, Shibata F, Oki T, Nakajima H, Nosaka T, Kumagai H (2003) Retrovirus-mediated gene transfer and expression cloning: powerful tools in functional genomics. Exp Hematol 31:1007-1014.

Klco JM, Lassere TB, Baranski TJ (2003) C5a receptor oligomerization: I. Disulfide trapping reveals oligomers and potential contact surfaces in a $G$ protein-coupled receptor. J Biol Chem 278:35345-35353.

Kornilova AY, Kim J, Laudon H, Wolfe MS (2006) Deducing the transmembrane domain organization of presenilin- 1 in $\gamma$-secretase by cysteine disulfide cross-linking. Biochemistry 45:7598-7604.

Li Y, Xu M, Lai M, Huang Q, Castro JL, Dimuzio-Mower J, Harrison T, Lellis C, Nadin A, Neduvelil JG, Register RB, Sardana MK, Shearman MS, Smith AL, Shi X, Yin K, Shafer JA, Gardell SJ (2000) Photoactivated $\gamma$-secretase inhibitors directed to the active site covalently label presenilin 1. Nature 405:689-694.

Lleó A, Berezovska O, Herl L, Raju S, Deng A, Bacskai BJ, Frosch MP, Irizarry 
M, Hyman BT (2004) Nonsteroidal anti-inflammatory drugs lower A $\beta 42$ and change presenilin 1 conformation. Nat Med 10:1065-1066.

Loo TW, Clarke DM (2001) Determining the dimensions of the drugbinding domain of human P-glycoprotein using thiol cross-linking compounds as molecular rulers. J Biol Chem 276:36877-36881.

Moehlmann T, Winkler E, Xia X, Edbauer D, Murrell J, Capell A, Kaether C, Zheng H, Ghetti B, Haass C, Steiner H (2002) Presenilin-1 mutations of leucine 166 equally affect the generation of the Notch and APP intracellular domains independent of their effect on A $\beta 42$ production. Proc Natl Acad Sci U S A 99:8025-8030.

Morohashi Y, Kan T, Tominari Y, Fuwa H, Okamura Y, Watanabe N, Sato C, Natsugari H, Fukuyama T, Iwatsubo T, Tomita T (2006) C-terminal fragment of presenilin is the molecular target of a dipeptidic $\gamma$-secretasespecific inhibitor DAPT (N-[N-(3,5-difluorophenacetyl)-L-alanyl]-Sphenylglycine $t$-butyl ester). J Biol Chem 281:14670-14676.

Ohki Y, Higo T, Uemura K, Shimada N, Osawa S, Berezovska O, Yokoshima S, Fukuyama T, Tomita T, Iwatsubo T (2011) Phenylpiperidine-type $\gamma$-secretase modulators target the transmembrane domain 1 of presenilin 1. EMBO J 30:4815-4824.

Page RM, Baumann K, Tomioka M, Pe BI, Fukumori A, Jacobsen H, Flohr A, Luebbers T, Ozmen L, Steiner H, Haass C (2008) Generation of A $\beta 38$ and $A \beta 42$ is independently and differentially affected by familial Alzheimer disease-associated presenilin mutations and $\gamma$-secretase modulation. J Biol Chem 283:677-683.

Park HK, Duk LN, Lee JH, Kim JW, Ki CS (2008) Identification of PSEN1 and APP gene mutations in Korean patients with early-onset Alzheimer's disease. J Korean Med Sci 23:213-217.

Pozdnyakov N, Murrey HE, Crump CJ, Pettersson M, Ballard TE, Am Ende CW, Ahn K, Li YM, Bales KR, Johnson DS (2013) $\gamma$-Secretase modulator (GSM) photoaffinity probes reveal distinct allosteric binding sites on presenilin. J Biol Chem 288:9710-9720.

Qi-Takahara Y, Morishima-Kawashima M, Yu T, Dolios G, Hirotani N, Horikoshi Y, Kametani F, Maeda M, Takaomi CS, Wang R, Ihara Y (2005) Longer forms of amyloid protein: implications for the mechanism of intramembrane cleavage by $\gamma$-secretase. J Neurosci 25:436-445.

Rogaeva EA, Fafel KC, Song YQ, Medeiros H, Sato C, Liang Y, Richard E, Rogaev EI, Frommelt P, Sadovnick AD, Meschino W, Rockwood K, Boss MA, Mayeux R, St George-Hyslop P (2001) Screening for PS1 mutations in a referral-based series of AD cases: 21 novel mutations. Neurology $57: 621-625$

Sato C, Morohashi Y, Tomita T, Iwatsubo T (2006) Structure of the catalytic pore of $\gamma$-secretase probed by the accessibility of substituted cysteins. J Neurosci 26:12081-12088.

Sato C, Takagi S, Tomita T, Iwatsubo T (2008) The C-terminal PAL motif and transmembrane domain 9 of presenilin 1 are involved in the formation of the catalytic pore of the $\gamma$-secretase. J Neurosci 28:6264-6271.

Shearman MS, Beher D, Clarke EE, Lewis HD, Harrison T, Hunt P, Nadin A, Smith AL, Stevenson G, Castro JL (2000) L-685,458, an aspartyl protease transition state mimic, is a potent inhibitor of amyloid $\beta$-protein precursor $\gamma$-secretase activity. Biochemistry 39:8698-8704.

Sodeyama N, Iwata T, Ishikawa K, Mizusawa H, Yamada M, Itoh Y, Otomo E, Matsushita M, Komatsuzaki Y (2001) Very early onset Alzheimer's disease with spastic paraparesis associated with a novel presenilin 1 mutation (Phe237Ile). J Neurol Neurosurg Psychiatry 71:556-557.

Soman KV, Karimi A, Case DA (1991) Unfolding of an $\alpha$-helix in water. Biopolymers 31:1351-1361.

Somavarapu AK, Kepp KP (2017) Membrane dynamics of $\gamma$-secretase provides a molecular basis for $\beta$-amyloid binding and processing. ACS Chem Neurosci 8:2424-2436.

Strisovsky K (2013) Structural and mechanistic principles of intramembrane proteolysis-lessons from rhomboids. FEBS J 280:1579-1603.

Takagi S, Tominaga A, Sato C, Tomita T, Iwatsubo T (2010) Participation of transmembrane domain 1 of presenilin 1 in the catalytic pore structure of the $\gamma$-secretase. J Neurosci 30:15943-15950.

Takagi-Niidome S, Osawa S, Tomita T, Iwatsubo T (2013) Inhibition of $\gamma$-secretase activity by a monoclonal antibody against the extracellular hydrophilic loop of presenilin 1. Biochemistry 52:61-69.

Takagi-Niidome S, Sasaki T, Osawa S, Sato T, Morishima K, Cai T, Iwatsubo T, Tomita T (2015) Cooperative roles of hydrophilic loop 1 and the C-terminus of presenilin 1 in the substrate-gating mechanism of $\gamma$-secretase. J Neurosci 35:2646-2656.
Takami M, Nagashima Y, Sano Y, Ishihara S, Morishima-Kawashima M, Funamoto S, Ihara Y (2009) $\gamma$-Secretase: successive tripeptide and tetrapeptide release from the transmembrane domain of $\beta$-carboxyl terminal fragment. J Neurosci 29:13042-13052.

Takasugi N, Tomita T, Hayashi I, Tsuruoka M, Niimura M, Takahashi Y, Thinakaran G, Iwatsubo T (2003) The role of presenilin cofactors in the $\gamma$-secretase complex. Nature 422:2114-2117.

Takeo K, Tanimura S, Shinoda T, Osawa S, Zahariev IK, Takegami N, Ishizuka-Katsura Y, Shinya N, Takagi-Niidome S, Tominaga A, Ohsawa N, Kimura-Someya T, Shirouzu M, Yokoshima S, Yokoyama S, Fukuyama T, Tomita T, Iwatsubo T (2014) Allosteric regulation of $\gamma$-secretase activity by a phenylimidazole-type $\gamma$-secretase modulator. Proc Natl Acad Sci U S A 111:10544-10549.

Ting SK, Benzinger T, Kepe V, Fagan A, Coppola G, Porter V, Hecimovic S, Chakravertyh S, Alvarez-Retuerto AI, Goate A, Ringman JM (2014) A novel PSEN1 mutation (I238M) associated with early-onset Alzheimer's disease in an African-American woman. J Alzheimers Dis 40:271-275.

Tominaga A, Cai T, Takagi-Niidome S, Iwatsubo T, Tomita T (2016) Conformational changes in transmembrane domain 4 of presenilin 1 are associated with altered amyloid- $\beta 42$ production. J Neurosci 36:1362-1372.

Tomita T (2014) Molecular mechanism of intramembrane proteolysis by $\gamma$-secretase. J Biochem 156:195-201.

Tomita T (2017) Probing the structure and function relationships of presenilin by substituted-cysteine accessibility method. Methods Enzymol 584:185-205.

Tomita T, Takikawa R, Koyama A, Morohashi Y, Takasugi N, Saido TC, Maruyama K, Iwatsubo T (1999) C Terminus of Presenilin Is Required for Overproduction of Amyloidogenic A $\beta 42$ through Stabilization and Endoproteolysis of Presenilin. J Neurosci 19:10627-10634.

Tomita T, Maruyama K, Saido TC, Kume H, Shinozaki K, Tokuhiro S, Capell A, Walter J, Grunberg J, Haass C, Iwatsubo T, Obata K (1997) The presenilin 2 mutation (N141I) linked to familial Alzheimer disease (Volga German families) increases the secretion of amyloid protein ending at the 42nd (or 43rd) residue. Proc Natl Acad Sci U S A 94:2025-2030.

Tomita T, Watabiki T, Takikawa R, Morohashi Y, Takasugi N, Kopan R, De Strooper B, Iwatsubo T (2001) The first proline of PALP motif at the C terminus of presenilins is obligatory for stabilization, complex formation, and $\gamma$-secretase activities of presenilins. J Biol Chem 276:33273-33281.

Uemura K, Lill CM, Li X, Peters JA, Ivanov A, Fan Z, De Strooper B, Bacskai BJ, Hyman BT, Berezovska O (2009) Allosteric modulation of PS $1 / \gamma$ secretase conformation correlates with amyloid $\beta 42 / 40$ ratio. PLoS One 4:e7893.

Watanabe N, Tomita T, Sato C, Kitamura T, Morohashi Y, Iwatsubo T (2005) Pen-2 is incorporated into the $\gamma$-secretase complex through binding to transmembrane domain 4 of presenilin 1. J Biol Chem 280:41967-41975.

Watanabe N, Takagi S, Tominaga A, Tomita T, Iwatsubo T (2010) Functional analysis of the transmembrane domains of presenilin 1: participation of transmembrane domains 2 and 6 in the formation of initial substrate-binding site of $\gamma$-secretase. J Biol Chem 285:19738-19746.

Wisniewski T, Dowjat WK, Buxbaum JD, Khorkova O, Efthimiopoulos S, Kulczycki J, Lojkowska W, Wegiel J, Wisniewski HM, Frangione B (1998) A novel Polish presenilin-1 mutation (P117L) is associated with familial Alzheimer's disease and leads to death as early as the age of 28 years. Neuroreport 9:217-221.

Xu X (2009) $\gamma$-Secretase catalyzes sequential cleavages of the A $\beta$ PP transmembrane domain. J Alzheimers Dis 16:211-224.

Yasuda M, Maeda K, Ikejiri Y, Kawamata T, Kuroda S, Tanaka C (1997) A novel missense mutation in the presenilin-1 gene in a familial Alzheimer's disease pedigree with abundant amyloid angiopathy. Neurosci Lett 232:29-32.

Zhao B, Yu M, Neitzel M, Marugg J, Jagodzinski J, Lee M, Hu K, Schenk D, Yednock T, Basi G (2008) Identification of $\gamma$-secretase inhibitor potency determinants. J Biol Chem 283:2927-2938.

Zhao G, Mao G, Tan J, Dong Y, Cui M, Kim S, Xu X (2004) Identification of a new presenilin-dependent $\zeta$-cleavage site within the transmembrane domain of amyloid precursor protein. J Biol Chem 279:50647-50651.

Zhou R, Yang G, Guo X, Zhou Q, Lei J, Shi Y (2019) Recognition of the amyloid precursor protein by human $\gamma$-secretase. Science 363:1-8.

Zhou Y, Moin SM, Urban S, Zhang Y (2012) An internal water-retention site in the rhomboid intramembrane protease GlpG ensures catalytic efficiency. Structure 20:1255-1263. 